OPEN ACCESS

Edited by:

Tianhui Chen,

University of Chinese Academy of

Sciences, China

Reviewed by:

Wenlong Lin,

Zhejiang University, China

Kongyang $\mathrm{Ma}$,

Sun Yat-Sen University, China

*Correspondence:

Liping Jin

jinlp01@163.com

Changgang Wang

changgangwang@gmail.com

${ }^{t}$ These authors have contributed equally to this work

Specialty section:

This article was submitted to

Molecular Diagnostics and

Therapeutics,

a section of the journal

Frontiers in Molecular Biosciences

Received: 28 March 2021 Accepted: 28 June 2021

Published: 09 July 2021

Citation:

Cai $D$, Wang $F$, Wang $C$ and Jin $L$

(2021) Phenotypic and Functional

Analyses of B7S1 in Ovarian Cancer.

Front. Mol. Biosci. 8:686803.

doi: 10.3389/fmolb.2021.686803

\section{Phenotypic and Functional Analyses of B7S1 in Ovarian Cancer}

\author{
Dongli Cai ${ }^{1+}$, Fang Wang ${ }^{2+}$, Changgang Wang ${ }^{3 *}$ and Liping Jin ${ }^{1 *}$ \\ ${ }^{1}$ Clinical and Translational Research Center, Shanghai First Maternity and Infant Hospital, School of Medicine,Tongji University, \\ Shanghai, China, ${ }^{2}$ Department of Gynaecology, Shanghai East Hospital, School of Medicine, Tongji University, Shanghai, China, \\ ${ }^{3}$ Department of General Surgery, Ruijin Hospital, Shanghai Jiao Tong University School of Medicine, Shanghai, China
}

Background: Although programmed death (PD) ligand 1 (PD-L1)/PD-1 inhibitors show potent and durable antitumor effects in a variety of tumors, their efficacy in patients with OvCa is modest. Thus, additional immunosuppressive mechanisms beyond PD-L1/PD-1 need to be identified.

Methods: The mRNA expression profiles of OvCa patients were obtained from The Cancer Genome Atlas (TCGA) database. The expression and clinical characteristics of VTCN1 (encoding B7S1) in OvCa were analyzed. The molecular interaction network, Gene Ontology (GO) analysis and Gene set enrichment analysis (GSEA) were used to functionally annotate and predict signaling pathways of VTCN1 in OvCa. Moreover, 32 treatment-naive patients with OvCa were recruited to assess B7S1 expression. The cytotoxic immune phenotypes in distinct subgroups were analyzed.

Results: B7S1 expression was increased in tumor sections compared with that in normal tissues from OvCa patients at both the mRNA and protein levels. VTCN1 expression was significantly correlated with the mRNA expression levels of several other co-inhibitory immune checkpoints. B7S1 protein was found to be highly expressed in $\mathrm{CD} 45^{+} \mathrm{CD} 68^{+}$ myeloid cells, whereas its putative receptor was expressed in $\mathrm{CD}^{+}$tumor-infiltrating lymphocytes (TILS). Furthermore, expression of B7S1 in antigen-presenting cells (APCs) was significantly correlated with the cytolytic function of $\mathrm{CD} 8^{+} \mathrm{TILs}$. Functional annotations indicated that VTCN1 was involved in regulating T cell-mediated immune responses and participated in the activation of a variety of classic signaling pathways related to the progression of human cancer.

Conclusion: In OvCa, B7S1 was highly expressed and may initiate dysfunction of CD8 ${ }^{+}$ TILs, which could be targeted for cancer immunotherapy.

Keywords: ovarian cancer, immune checkpoint, B7S1, tumor-infiltrating lymphocytes, immunotherapy

\section{INTRODUCTION}

Ovarian cancer $(\mathrm{OvCa})$ is one of the most lethal gynecologic malignancies, with increasing global incidence. Global Cancer Statistics estimated that over 295,414 new OvCa cases and 184,799 OvCarelated deaths occurred worldwide in 2018 (Bray et al., 2018). Owing primarily to the lack of effective screening strategies and the absence of early specific symptoms, more than $75 \%$ patients with OvCa present disease stage III or IV at diagnosis (Hung et al., 2016), thereby contributing to a poor overall survival. Although aggressive frontline treatments with taxane/platinum-based chemotherapy and 
cytoreductive surgery, more than $70 \%$ of patients with advancedstage cancer relapse within 5 years and become resistant to chemotherapy; the 5-year overall survival rate remains ominously low at 45\% (Capriglione et al., 2017). Thus, there is an urgent need for the development of novel therapeutics for advanced OvCa.

Recent studies have shown that, like many solid tumors, OvCa is immunogenic (Zhang et al., 2003) and can elicit a spontaneous antitumor immune response (Cai et al., 2020). Engaging the immune system is a critical component of optimal $\mathrm{OvCa}$ therapy, and the quality of tumor-infiltrating T cells (TILs) is a critical determinant of outcomes in patients with OvCa (Zhang et al., 2003; Hamanishi et al., 2007; Hwang et al., 2012). T cellmediated immune responses are regulated by several costimulatory and co-inhibitory signals (Sun et al., 2018). Evidence in several cancer systems has shown that inhibitory immune checkpoint receptors expressed on $\mathrm{T}$ cells promote T-cell exhaustion, dampen host immunity, and assist tumor evasion (Thommen et al., 2018). Blockade of these inhibitory checkpoint receptors with specific antibodies has been reported to reinstate antitumor responses at different levels (Pardoll, 2012).

Programmed death (PD)-1 is a prototypical co-inhibitory checkpoint receptor that has emerged as a critical intrinsic modulatory mechanism for impairing natural antitumor immunity (Gaillard et al., 2016). Interfering with PD-1 or its ligand PD-L1 has been shown to enhance antineoplastic immune responses through the recovery of $\mathrm{T}$-cell function in a wide spectrum of tumors (Mariathasan et al., 2018), including melanoma (Patel and Kurzrock, 2015), non-small cell lung cancer (Patel and Kurzrock, 2015) and renal cell carcinomas (McDermott et al., 2016). However, although the PD-1/PD-L1 pathway blockade produces durable clinical responses in preclinical tests for OvCa, the best overall response rate in 26 PD-L1-positive patients treated with pembrolizumab (anti-PD-1) was only $11.5 \%$ (Disis et al., 10718). Since immune checkpoint molecules function nonredundantly and cooperatively to finetune immune responses and promote T-cell exhaustion ( $\mathrm{Li}$ et al., 2018), additional immunosuppressive mechanisms need to be identified.

B7S1 (VTCN1, B7-H4, B7x) is a member of the B7 superfamily and shows $25 \%$ amino acid identity with other B7 family members (Hansen et al., 2009). B7S1 mRNA is broadly expressed; however, the restricted pattern of protein expression in normal tissue suggests post-transcriptional regulation (Choi et al., 2003). Aberrant B7S1 expression has been reported in a vast majority of human malignancies, including melanoma (Quandt et al., 2011), renal cell carcinoma (Krambeck et al., 2006), breast cancer (Tringler et al., 2005), non-small cell lung cancer (Sun et al., 2006), thyroid cancer (Zhu et al., 2013), and hepatocellular carcinoma (Kang et al., 2017). B7S1 expression in multiple solid tumors is positively correlated with malignant phenotypes, such as advanced lymph node metastasis, high tumor stage, poor differentiation, early recurrence, and is reversely related to the infiltration intensity of TILs, as well as the overall survival rate (Wang and Wang, 2020).
B7S1 expression is restricted to antigen-presenting cells (APCs), and its putative receptor is induced on activated $\mathrm{T}$ cells to inhibit their proliferation, cytokine production, and cytotoxicity (Kryczek et al., 2006; Kryczek et al., 2007; Li et al., 2018). In human hepatocellular carcinoma, B7S1 expression is reportedly upregulated on APCs, and its putative receptor is coexpressed with PD-1 on activated early CD8 ${ }^{+}$TILs, to promote $\mathrm{T}$ cell exhaustion and depress anti-tumor immune response via upregulating Eomes ( $\mathrm{Li}$ et al., 2018). Studies have shown that B7S1 is closely relevant to classical signaling pathways. In esophageal squamous cell carcinoma, by activating JAK2/ STAT3 pathway, B7S1 stimulated the secretion of IL-6, which in turn upregulated the expression of B7S1, thereby mutually enhancing the growth and tumorigenicity of cancer cells (Chen et al., 2016). Incubated T cells with B7S1-Ig fusion protein in vitro significantly inhibits the proliferation and cytotoxic activity of $\mathrm{T}$ cells, by interfering with the activation of ERK, JNK, and AKT (Wang et al., 2012; Wang and Wang, 2020). B7S1 silencing enhanced drug-induced apoptosis by inhibiting the PTEN/ $\mathrm{PI} 3 \mathrm{~K} / \mathrm{AKT}$ pathway in triple-negative breast cancer cells (Wang et al., 2018). Furthermore, blockade of B7S1 downregulated the transcription of CXCL12/CXCR4. By activating ERK1/2, AKT, PI3K and the other signaling pathways, CXCL12/CXCR4 axis is widely involved in the proliferation, invasion and metastasis of tumor cells (Peng et al., 2015).

B7S1 is highly expressed in primary and metastatic serous, endometrioid, clear cell and epithelial ovarian carcinomas, but is low in mucinous and non-epithelial ovarian carcinomas (Wang and Wang, 2020). According to a recent study, B7S1 was predominantly expressed by ovarian cancer cells, and this alteration is positively correlated with the expression of C-X-C motif chemokine ligand 17 and the proportion of infiltrating mature APCs (MacGregor et al., 2019). However, in 2006, a suppressive macrophage population with B7S1 expression has been detected in human ovarian carcinoma. The $\mathrm{B} \mathrm{S1}^{+}$ macrophages express CD86 at a higher level and have stronger inhibitory activity than $\mathrm{B}^{-} \mathrm{S}^{-}$macrophages (Kryczek et al., 2006; Kryczek et al., 2007). Due to high expression level of B7S1 in OvCa was found significantly associated with tumor stage (Liang et al., 2016) and a worse progression-free survival (PFS) (Ye et al., 2018), B7S1 may serve as a promising candidate target for OvCa immunotherapy.

Accordingly, in this study, we aimed to determine the expression and roles of $\mathrm{B} 7 \mathrm{~S} 1$ in $\mathrm{OvCa}$, with a focus on its relation to cancer-associated immune responses. Our findings demonstrated that B7S1 suppressed antitumor immunity and supported the applications of B7S1 as a promising target for immunotherapy in OvCa.

\section{MATERIALS AND METHODS}

\section{Analysis of the Public Dataset}

RNA sequencing-based gene expression data of samples from patients with OvCa were obtained from Gene Expression Profiling for Interactive Analysis (GEPIA) for Cancer 
Genomics (http://gepia.cancer-pku.cn/) (Tang et al., 2017) and TISIDB (http://cis.hku.hk/TISIDB/) (Ru et al., 2019).

\section{Human Specimens}

Fresh tumor tissues, malignant ascites, and matched blood were collected from 32 patients with OvCa undergoing primary surgical treatment without chemotherapy at Shanghai First Maternity and Infant Hospital. All experimental protocols were approved by the Ethical Committee of the Shanghai First Maternity and Infant Hospital (IEC approval NO. 2017-100), and informed consent was obtained from patients prior to their enrollment in the study.

\section{Isolation of Peripheral Blood Mononuclear Cells and TILs from Tumors or Ascites}

Blood and ascites from patients with OvCa were drawn into heparinized tubes and centrifuged on Ficoll-Hypaque density gradients (cat. no. 17-1440-02; GE Healthcare Life Sciences). Fresh tumor tissues from patients with OvCa were digested in RPMI-1640 medium supplemented with $1 \mathrm{mg} / \mathrm{ml}$ collagenase IV (cat. no. 17104019; Gibco) for $30 \mathrm{~min}$ at $37^{\circ} \mathrm{C}$ prior to FicollHypaque density gradient centrifugation. This method has been described previously (5).

\section{Immunofluorescence}

Paraffin sections of human OvCa specimens were dewaxed in xylene, dehydrated in ethanol, subjected to heat-induced epitope retrieval, and then incubated with primary antibodies against human CD45 (cat. no. ab10559; Abcam, Cambridge, United Kingdom) and B7S1 (MIH43; cat. no. ab110221; Abcam, Cambridge, United Kingdom) at $4^{\circ} \mathrm{C}$ overnight. AffiniPure F (ab')2 Fragment donkey anti-rabbit immunoglobulin (cat. no. 711-546-152; Jackson Immuno Research, West Grove, PA, United States) and AffiniPure F (ab')2 Fragment donkey anti-mouse immunoglobulin (cat. no. 715-166-150; Jackson Immuno Research, West Grove, PA, United States) were chosen as the secondary antibodies. All slides were incubated with mounting medium containing $4^{\prime}, 6-$ diamidino-2-phenylindole for $20 \mathrm{~min}$. Images were obtained using a Zeiss fluorescence microscope. Quantification analysis was performed using ImageJ software (National Institutes of Health, Bethesda, MD, United States). The method has been described previously (Cai et al., 2020).

\section{Flow Cytometry}

The following fluorescent dye-conjugated antibodies were used: anti-CD45-PerCP-CY5.5 (HI30; cat. no.304028, Biolegend), antilineage-fluorescein isothiocyanate-FITC (UCHT1, HCD14, 3G8, HIB19, 2H7, HCD56; cat. no. 348701, Biolegend), anti-human leukocyte antigen (HLA)-DR-AF700 (L243; cat. no. 307626Biolegend), anti-CD14-allophycocyanin (APC)-CY7 (M4P9; cat. no. 557831, BD), anti-CD15-phycoerythrin (PE)CY5 (W6D3; cat. no. 323014, Biolegend), anti-B7S1-PE-CY7 (MIH43; cat. no. 358106, Biolegend), anti-CD3-PerCP-CY5.5 (OKT3; cat. no. 300328, Biolegend), anti-CD56-FITC (HCD56; cat. no. 318304, Biolegend), anti-CD4-PE-CF594 (L200; cat. no.
562402, BD), anti-CD8-AF700 (SK1; BD), anti-FoxP3-PE-CF594 (259D/C7; cat. no. 563955, BD), anti-T cell immunoglobulin and mucin domain-containing protein 3 (TIM-3)-PE (F38-2E2; cat. no. 345006, Biolegend), anti-CD27-PerCP-CY5.5 (O323; cat. no. 302820, Biolegend), anti-Ki-67-AF700 (B56; BD), anti-tumor necrosis factor (TNF)- $\alpha$-PE-CF594 (Mab11; cat. no. 502946, Biolegend), and anti-interferon (IFN)- $\gamma$-PE-CY7 (B27; cat. no. 506518, Biolegend). Dead cells were excluded by viability dye staining (Fixable viability dye eF506; cat. no. 65-0866-18, eBioscience), as described previously (Cai et al., 2020).

To detect the expression of human B7S1 receptor, cell suspensions were incubated with $10 \mu \mathrm{g} / \mathrm{ml}$ biotin-labeled human B7S1-mouse IgG2a Fc fusion protein (generated inhouse) at $4^{\circ} \mathrm{C}$ for $40 \mathrm{~min}$ and then with $0.5 \mu \mathrm{g} / \mathrm{ml}$ streptavidin Brilliant Violet 421 (cat. no. 405225 Biolegend) together with surface antibodies. Cells were acquired using an LSRFortessa flow cytometer, and data were analyzed using FlowJo.X software (Tree Star, Ashland, OR, United States).

\section{Protein-Protein Interaction (PPI) Network Construction}

The STRING (https://string-db.org/) online database was used to analyze the functional interactions between proteins. Cytoscape (version 3.5) was employed to visualize the molecular interaction network.

\section{Functional Annotations}

mRNA expression profiles of OvCa were obtained from The Cancer Genome Atlas (TCGA, https://cancergenome.nih.gov/) online database. Gene Ontology (GO) enrichment analysis for hub genes was performed and visualized using ClueGo (version 2. 5.7) and CluePedia (version 1.5.7). Gene set enrichment analysis (GSEA) was used to predict potential hallmarks of VTCN1 in OvCa.

\section{Statistics}

Statistical analyses were conducted with Prism 6.0 software (GraphPad), using the appropriate tests as indicated in the legends. All values are expressed as means \pm standard errors of the means. Results with $p$ values less than 0.05 were considered statistically significant.

\section{RESULTS}

\section{VTCN1 was Highly Expressed in Human OvCa and was Correlated With Multiple Co-inhibitory Immune Checkpoint Genes}

The low response rate to $\mathrm{PD}-1$ blockade in $\mathrm{OvCa}$ may be associated with co-expression of other co-inhibitory immune checkpoint molecules in the tumor microenvironment (TME). To identify potential targets in patients with $\mathrm{OvCa}$, we examined the mRNA expression levels of several checkpoint molecules in various tumors. RNA-sequencing data from TCGA revealed high upregulation of VTCN1 (encoding B7S1) in several solid tumors, 

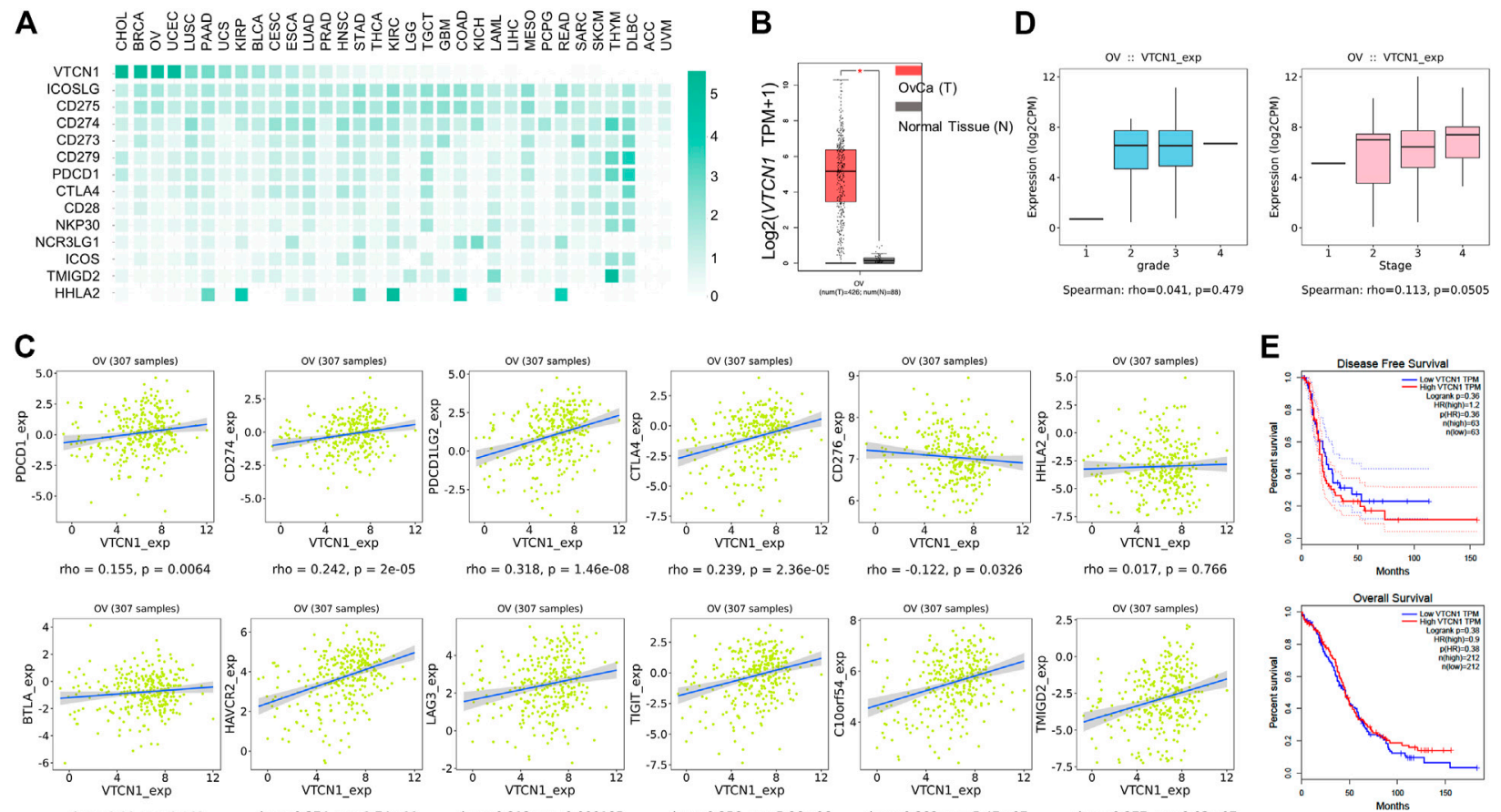

rho $=0.08, p=0.163$

rho $=0.374, p=1.74 \mathrm{e}-11$

rho $=0.212, p=0.000185$

rho $=0.256, p=5.86 \mathrm{e}-0$

rho $=0.282, p=5.47 \mathrm{e}-0$
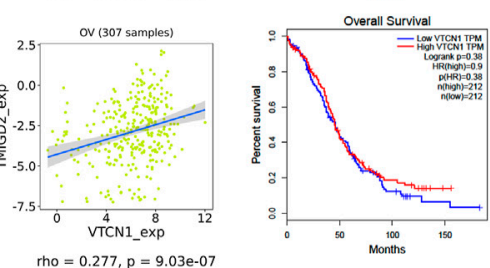

FIGURE 1 | VTCN1 was overexpressed in OvCa and correlated with multiple co-inhibitory immune checkpoint genes. (A) Heatmap analysis of the mRNA expression of B7/CD28 family genes in various human tumors, shown as scaled log2-fold changes (GEPIA data). (B) The expression levels of $V T C N 1$ in human OvCa and normal controls. The data were derived from TCGA database and are shown on a log2 (TPM +1$)$ scale. TPM: transcripts per million. The $p$ value cutoff was 0.01 . ${ }^{*} p<0.05$. (C) Correlations of VTCN1 expression with tumor grade (left) and stage (right) in patients with OvCa. (D) Correlations of VTCN1 expression with the mRNA expression levels of several immune checkpoint proteins. (E) Correlations of VTCN1 expression with disease-free survival (upper panel) and overall survival (bottom panel) in patients with OvCa.

including human OvCa, as compared with known B7/CD28 family members (Figure 1A). Upregulation of $V T C N 1$, i.e., a $\log _{2}(\mathrm{TPM}+1)$ fold increase of more than 32, was observed in OvCa compared with normal tissues (Figure 1B). Moreover, the expression of VTCN1 was significantly positively correlated with the expression of some other checkpoint genes, such as CTLA4, HAVCR2, LAG3, TIGIT, and C10orf54 (Figure 1C). In addition, VTCN1 expression was not significantly correlated with tumor grade or tumor stage (Figure 1D). Stratification of patients with OvCa by VTCN1 expression revealed no significant differences in disease-free survival or overall survival, although patients with high VTCN1 expression had a high hazard ratio (HR; HR = 1.2) for relapse (Figure 1E). The highly expressed B7S1 in OvCa has been reported significantly associated with tumor stage (Liang et al., 2016) and a worse progression-free survival (Ye et al., 2018). The inconsistency between mRNA level and protein level may be related to the complex mechanism of post-transcriptional regulation. Taken together, these results implied that B7S1 may contribute to antitumor immunosuppression in OvCa.

\section{B7S1 Protein Was Mainly Expressed by Intratumoral Myeloid Cells in Human OvCa}

Because VTCN1 is differentially expressed in OvCa subtypes (Chen et al., 2018), we next evaluated the protein levels of B7S1 in tumor samples from primary debulking surgeries of
32 treatment-naïve patients with epithelial OvCa (Table. 1) by immunofluorescence and flow cytometry (Cai et al., 2020). Compared with normal ovary tissues, B7S1 was upregulated in tumor sections, as detailed in our previous study (Cai et al., 2020). Contrary to reported data demonstrating that B7S1 expression is restricted to the tumor cell compartment (MacGregor et al., 2019), our study showed that B7S1 was expressed on both $\mathrm{CD}^{-} 5^{-}$and $\mathrm{CD} 45^{+}$cells (Figure 2A, indicated by the white arrows). In $\mathrm{CD} 45^{+}$cells, B7S1 was predominantly detected within the $\mathrm{CD}^{+} 8^{+}$myeloid compartment (Figure 2A).

Given that B7S1 could be detected on tumor-infiltrating $\mathrm{CD} 45^{+}$cells, we sought to identify which subsets of myeloid cells expressed this target. TILs from tumor tissues and tumorassociated lymphocytes from ascites were isolated by densitygradient centrifugation. PBMCs from the same patients were used as controls. B7S1 was predominantly expressed on myeloid dendritic cells (mDCs), CD14 HLA-DR ${ }^{\text {hi }}$ monocytes/ macrophages $(\mathrm{Mø} / \mathrm{Mo})$, and $\mathrm{CD} 14^{+} \mathrm{HLA}-\mathrm{DR}^{\text {low/- }}$ monocytic myeloid-derived suppressed cells (mMDSCs). The percentages of B7S1-expressing APCs in the TME and ascites were significantly higher than those in peripheral blood (Figures 2B,C). We did not find a significant difference in B7S1 expression on $\mathrm{CD}_{15} 5^{+}$HLA-DR ${ }^{\text {low/- }}$ granulocytic myeloidderived suppressed cells (gMDSCs) among tumors, ascites, and PBMCs (Figures 2B,C). 
TABLE 1 | Clinical and pathological characteristics of patients with OvCa.

\begin{tabular}{|c|c|c|c|c|}
\hline Patient no & Age & FIGO Stage & Pathology & Chemotherapy \\
\hline P\#1 & 32 & IV & Low-grade adenocarcinoma & No \\
\hline P\#2 & 68 & Illc & Low-grade adenocarcinoma & No \\
\hline P\#3 & 59 & IIIC & Endometrioid adenocarcinoma & No \\
\hline P\#4 & 55 & $\| l l b$ & Low-grade adenocarcinoma & No \\
\hline P\#5 & 50 & IIIC & Low-grade adenocarcinoma & No \\
\hline P\#6 & 42 & IV & Serous papillary adenocarcinoma & No \\
\hline P\#7 & 54 & Ilc & Endometrioid adenocarcinoma & No \\
\hline P\#8 & 63 & Ila & High-grade adenocarcinoma & No \\
\hline P\#9 & 28 & Illc & Mucinous adenocarcinoma & No \\
\hline P\#10 & 51 & lc & Adenocarcinoma & No \\
\hline P\#11 & 54 & Ila & Endometrioid adenocarcinoma & No \\
\hline P\#12 & 45 & lc & Adenocarcinoma & No \\
\hline P\#13 & 67 & IIla & High-grade adenocarcinoma & No \\
\hline P\#14 & 48 & IIIC & High-grade adenocarcinoma & No \\
\hline P\#15 & 49 & IIIC & High-grade adenocarcinoma & No \\
\hline P\#16 & 54 & Illc & Low-grade adenocarcinoma & No \\
\hline P\#17 & 61 & Ila & Endometrioid adenocarcinoma & No \\
\hline P\#18 & 60 & - & Low-grade adenocarcinoma & No \\
\hline P\#19 & 67 & $\| l b$ & High-grade adenocarcinoma & No \\
\hline P\#20 & 39 & la & Mucinous carcinoma & No \\
\hline P\#21 & 66 & $\| l b$ & High-grade adenocarcinoma & No \\
\hline P\#22 & 60 & Ila & High-grade adenocarcinoma & No \\
\hline P\#23 & 48 & $\mathrm{lb}$ & Adenocarcinoma & No \\
\hline P\#24 & 61 & IIIC & High-grade adenocarcinoma & No \\
\hline P\#25 & 55 & - & Adenocarcinoma & No \\
\hline P\#26 & 51 & Ilc & Adenocarcinoma & No \\
\hline P\#27 & - & - & High-grade serous carcinoma & No \\
\hline P\#28 & 67 & llb & Adenocarcinoma & No \\
\hline P\#29 & 49 & IIIC & High-grade adenocarcinoma & No \\
\hline P\#30 & 51 & Illb & Low-grade adenocarcinoma & No \\
\hline P\#31 & 36 & la & Clear cell carcinoma & No \\
\hline P\#32 & 46 & $\|_{\mathrm{C}}$ & High-grade serous carcinoma & No \\
\hline
\end{tabular}

$\mathrm{B} 7 \mathrm{~S} 1$ is a negative immune checkpoint protein that binds to activated $\mathrm{T}$ cells and inhibits their proliferation and function (Li et al., 2018). To identify the potential targets of B7S1 in OvCa, we utilized a biotin-labeled human B7S1mouse IgG2a Fc fusion protein to assess the expression of the putative B7S1 receptor ( $\mathrm{pB} 7 \mathrm{~S} 1 \mathrm{R})$. $\mathrm{pB} 7 \mathrm{~S} 1 \mathrm{R}$ was detected on $\mathrm{CD}^{+} \mathrm{T}, \mathrm{CD} 8^{+} \mathrm{T}$, natural killer $(\mathrm{NK})$, and NK T cells in tumor tissues (Figures 2D,E). Because B7S1 expressed by tumorinfiltrating myeloid cells has been reported to induce dysfunction of antitumor $\mathrm{CD}^{+} \mathrm{T}$ cells in liver cancer ( $\mathrm{Li}$ et al., 2018), the expression patterns of B7S1 and pB7S1R strongly suggested that B7S1 expression in OvCa may inhibit $\mathrm{CD}^{+} \mathrm{T}$ cell function.

\section{B7S1 Expression was Inversely Correlated With the Cytolytic Function of CD8 ${ }^{+}$TILs}

To investigate the relevance of $\mathrm{B} 7 \mathrm{~S} 1$ expression in the infiltration and function of $\mathrm{CD}^{+}$TILs, patients with $\mathrm{OvCa}$ were divided into two groups (B7S1 ${ }^{\text {hi }}$ and $\mathrm{B} 7 \mathrm{~S} 1^{\text {low }}$ ) based on the average frequency of $\mathrm{B} 7 \mathrm{~S}^{+}$cells in all $\mathrm{CD} 14^{+}$myeloid cells (Hong et al., 2019) (cutoff $=22.9 \%$ ). Compared with $\mathrm{B} 7 \mathrm{~S} 1^{\text {hi }}$ patients, significantly lower frequencies of $\mathrm{CD} 4^{+}$ $\mathrm{T}$ cells and $\mathrm{CD} 4^{+}$Foxp $3^{+}$cells were found in $\mathrm{B} 7 \mathrm{~S} 1^{\text {low }}$ patients. In contrast, B7S $1^{\text {low }}$ patients had markedly higher fractions of $\mathrm{CD}^{+}$TILs and $\mathrm{CD} 8 / \mathrm{CD} 4$ ratios (Figures 3A,B). Moreover, $\mathrm{CD}^{+}$TILs isolated from $\mathrm{B} 7 \mathrm{~S} 1^{\text {hi }}$ patients displayed the characteristic exhausted $\mathrm{T}$-cell phenotypes, including higher levels of the co-inhibitory molecule PD-1, decreased surface expression of the co-stimulatory molecule CD27 and proliferation marker Ki67, and decreased levels of TNF- $\alpha$ upon phorbol myristate acetate/ionomycin stimulation in comparison with those from $\mathrm{B} 7 \mathrm{~S} 1^{\text {low }}$ patients (Figures 3C,D). $\mathrm{CD}^{+}$TILs isolated from $\mathrm{B} 7 \mathrm{~S} 1^{\text {hi }}$ patients tended to show increased TIM-3 expression (Figures 3C,D); the lack of a significant difference between the two groups may have been related to the small sample size in this study. In addition, although there was no statistically significant difference in the proportion of IFN $-\gamma^{+}$CD8 TILs (data not shown) between the two groups, which may be due to the lack of NK cells in some patients, the frequency of TNF- $\alpha^{+}$IFN- $\gamma^{+}$CD8 TILs was found to be increased in $\mathrm{B} 7 \mathrm{~S} 1^{\text {low }}$ patients (Figures $3 \mathrm{C}, \mathrm{D}$ ). Since almost half of $\mathrm{CD} 4{ }^{+} \mathrm{T}$ cells expressed $\mathrm{pB} 7 \mathrm{~S} 1 \mathrm{R}$ as shown in Figure 1D, we also analyzed the expression of PD-1 and IFN- $\gamma$ in CD $4^{+}$TILs. It was found that there were no obvious differences between $\mathrm{B} 7 \mathrm{~S} 1^{\text {hi }}$ and $\mathrm{B} 7 \mathrm{~S} 1^{\text {low }}$ groups (Figure $3 \mathrm{E}$ ), which could be due to the limited sample size. Collectively, these results indicated that B7S1 expression on APCs was inversely correlated with the infiltration and cytolytic function of $\mathrm{CD}^{+}$TILs in OvCa. 

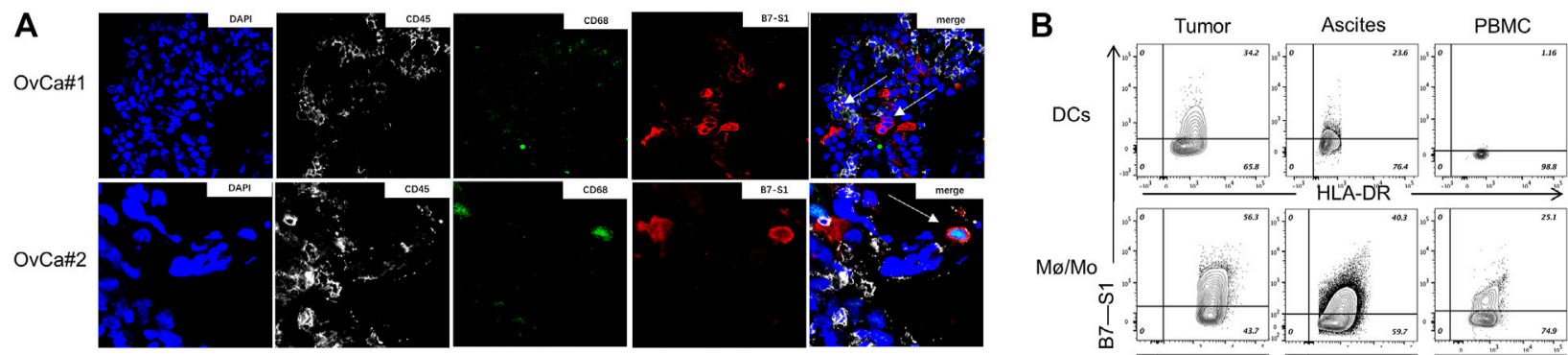

C

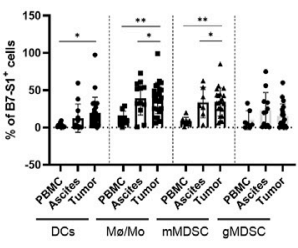

D

Gated on Live CD45+cells
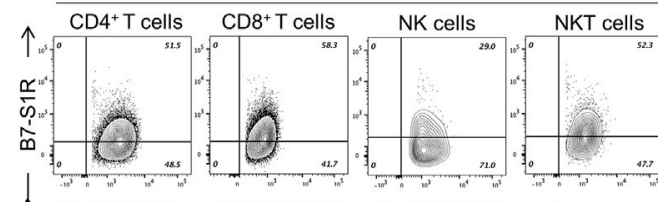

$\mathbf{E}$
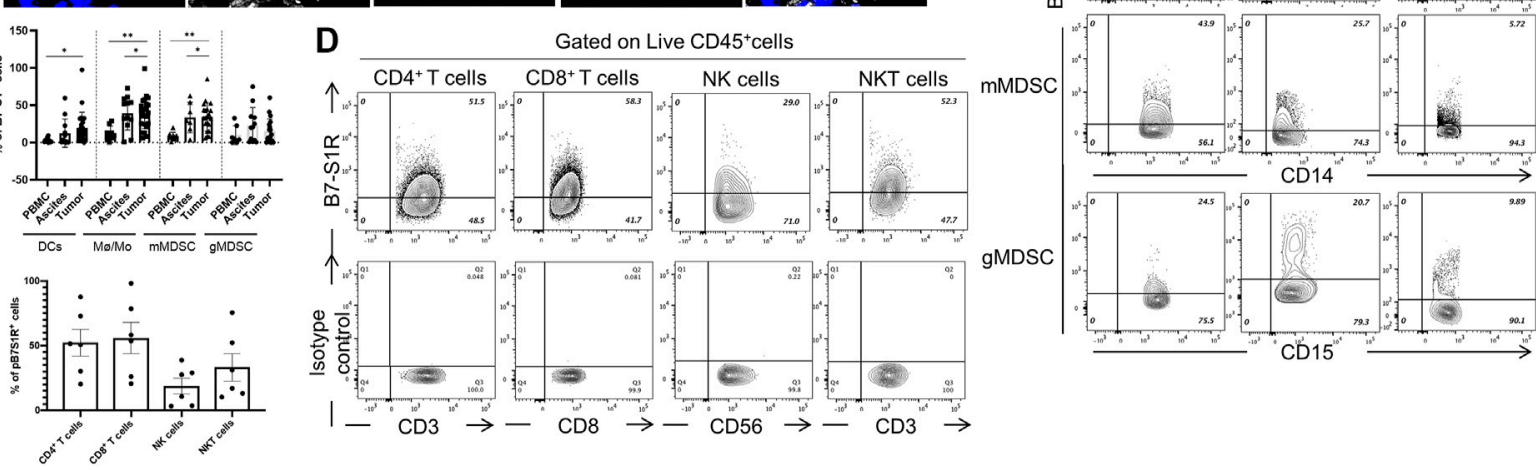

FIGURE 2 |B7S1 protein was mainly expressed in intratumoral myeloid cells in human OvCa. (A) Representative immunofluorescence images of B7S1 in human OvCa. Original magnification: 40x (OvCa\#1), 100x(OvCa\#2). (B,C) Representative figures and summarized data showing the percentages of B7S1-positive DCs, Mø/ Mo, mMDSCs, and gMDSCs in tumors, ascites, and PBMCs from patients with OvCa. Statistical analysis was performed using one-way analysis of variance followed by Tukey's multiple comparison tests. The data are presented as means \pm standard errors of the means; ${ }^{\text {ns }} P>0.05,{ }^{*} p<0.05,{ }^{* *} p<0.01$, and ${ }^{\star \star *} p<0.001$. (D,E) Representative figures and summarized data of pB7S1R expression on CD4 ${ }^{+} \mathrm{T}$ cells, CD8 ${ }^{+} \mathrm{T}$ cells, NK cells, and NK T cells in OvCa tumors detected by a biotinylated hB7S1-mlgG2a Fc fusion protein.

A

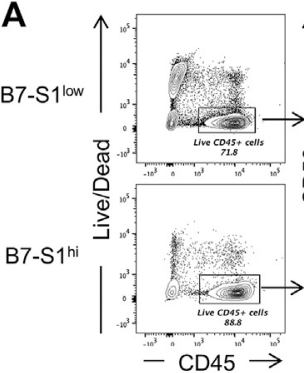

C

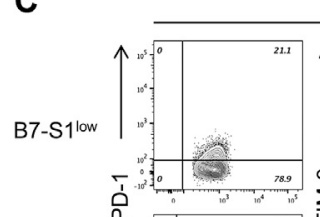

B7-S1

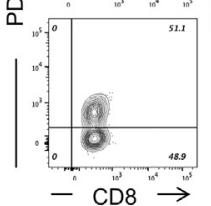

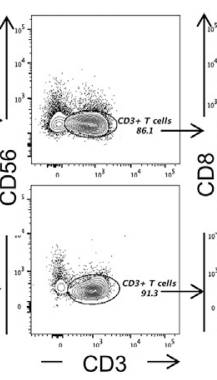

Gated on Live $\mathrm{CD} 45^{+} \mathrm{CD} 3^{+} \mathrm{CD} 8^{+} \mathrm{T}$ cells

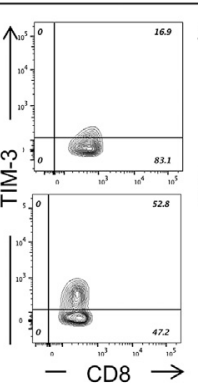

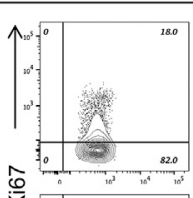

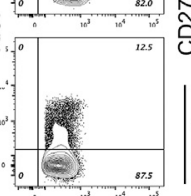

$\div \mathrm{CD} 8 \stackrel{10,5}{\rightarrow}$
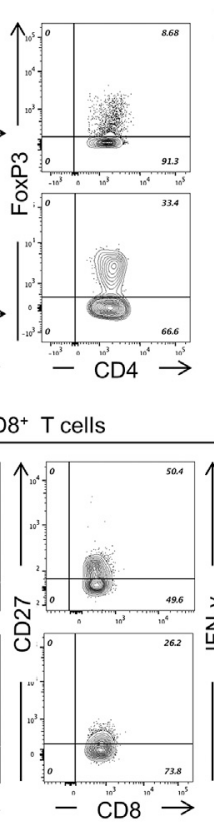
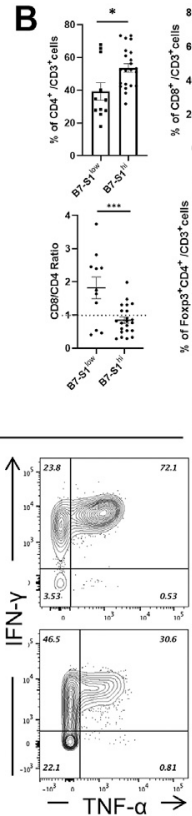
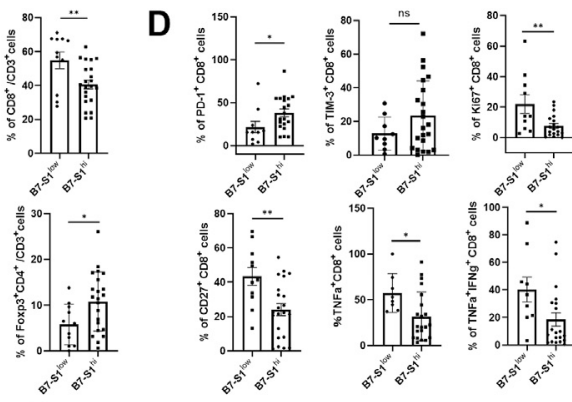

E Gated on Live $\mathrm{CD} 45^{+} \mathrm{CD} 3^{+} \mathrm{CD} 4^{+} \mathrm{T}$ cells
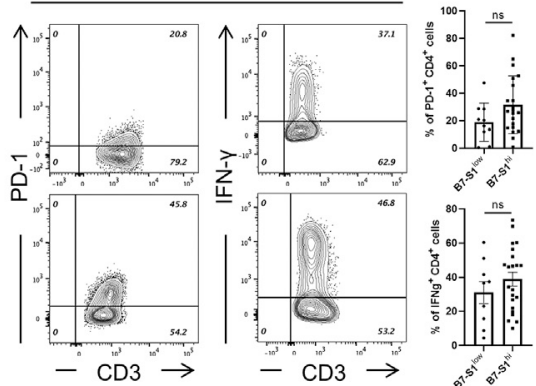

FIGURE 3 | B7S1 expression was inversely correlated with the cytolytic function of CD8+ TILs. (A,B) Representative figures and summarized data of infiltrated $\mathrm{T}$ cells in tumor tissues of patients with OvCa in B7S $1^{\text {hi }}$ and B7S $1^{\text {low }}$ groups. (C,D) Representative figures and summarized data displaying PD-1, Ki67, CD27, TNF $\alpha$, and IFN $\gamma$ expression in CD8 ${ }^{+} \mathrm{T}$ cells in tumors. (E) Representative figures and summarized data displaying PD-1 and IFN $\gamma$ expression in CD4 ${ }^{+} \mathrm{T}$ cells in tumors. The data are presented as means \pm standard errors of the means; $p$ value was computed by $t$-test. ${ }^{\text {ns }} P>0.05,{ }^{*} p<0.05,{ }^{\star *} p<0.01$, and ${ }^{\star \star \star} p<0.001$. 


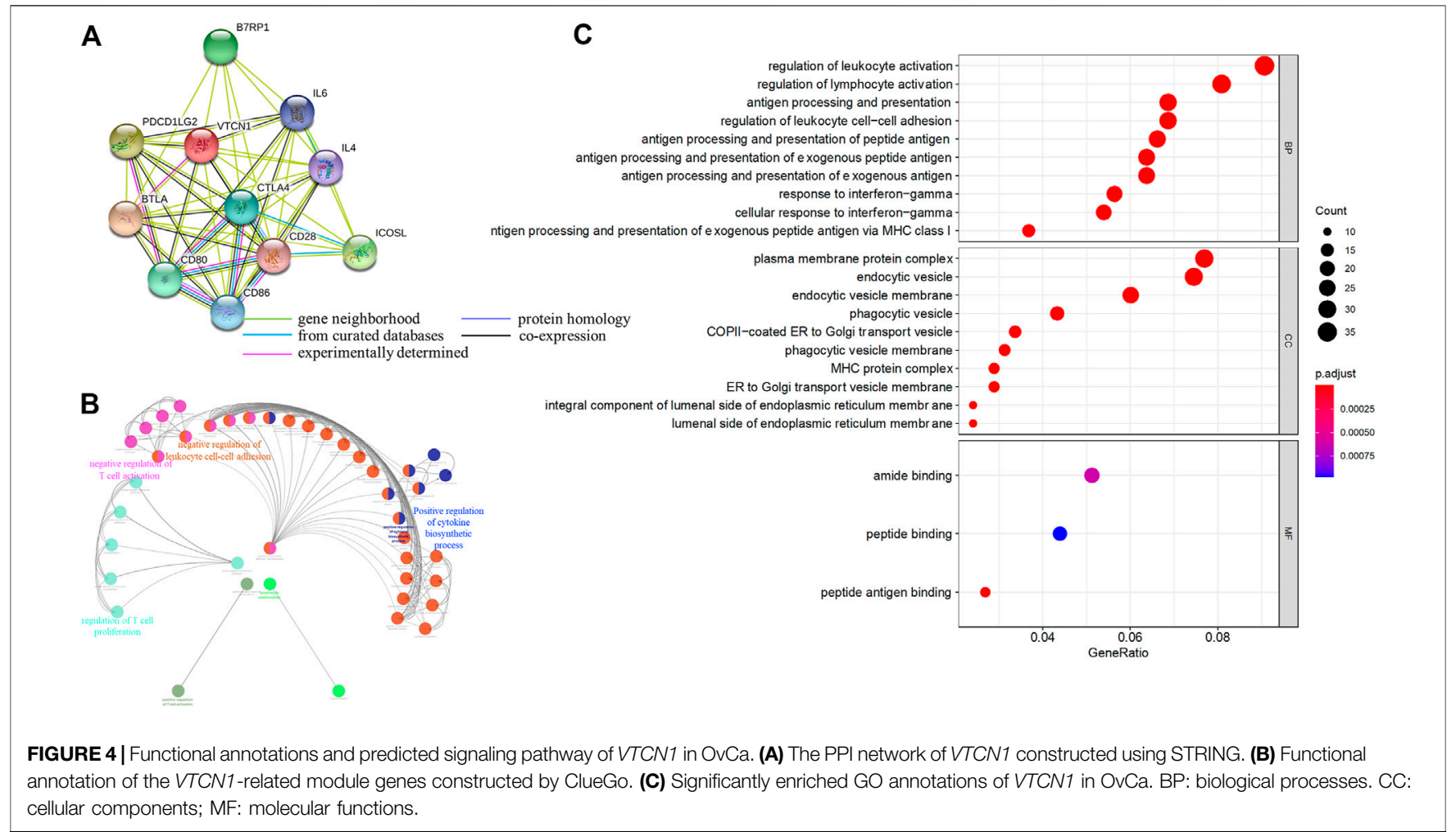

\section{Functional Annotations and Predicted Signaling Pathways}

Next, we systematically analyzed the biological functions of VTCN1 in OvCa. A PPI network of potential targets was constructed (Figure 4A), and the most significant modules were acquired using Cytoscape (Figure 4B). Functional analyses of VTCN1 demonstrated that genes in this module were mainly enriched in the regulation of $\mathrm{T}$-cell activation, T-cell proliferation, leukocyte cell-cell adhesion and cytokine biosynthetic process (Figure 4B). We selected genes that were co-expressed with VTCN1 in OvCa from TCGA database, and the top $500(p<0.01$ and correlation $>0.3)$ positively and negatively correlated genes were processed via $\mathrm{GO}$ functional analysis using the cluster-filer package of R software (Yu et al., 2012).We found that the significant GO terms in the biological process category were tightly associated with the regulation of lymphocyte activation, antigen processing and presentation, and response to IFN- $\gamma$ (Figure 4C).

To identify the VTCN1-related pathways activated in OvCa, we conducted GSEA between low and high VTCN1 expression datasets. Significant differences (false-discovery rate $<0.25, p<$ 0.05 ) in the enrichment of the Molecular Signature database Collection are shown in Figure 5. The results showed that VTCN1 was mainly involved in IL-2/signal transducer and activator of transcription (STAT)5 signaling, p53 pathway, mammalian target of rapamycin complex 1 (mTORC1) signaling, apoptosis, TNF- $\alpha$ signaling via nuclear factor (NF)$\kappa \mathrm{B}$, inflammatory response, IFN- $\gamma$ response, IFN- $\alpha$ response, IL6/Janus kinase (JAK)/STAT3 signaling, reactive oxygen species signaling, WNT/ $\beta$-catenin signaling, and KRAS signaling. These results showed that high expression of VTCN1 was closely associated with antitumor immune responses and malignancy in OvCa.

\section{DISCUSSION}

In this study, we demonstrated that B7S1 was highly expressed in OvCa tumor tissues compared with that in non-tumor tissues at both the mRNA and protein levels. B7S1 was mainly expressed by tumor-infiltrating APCs, and its putative receptor was expressed by $\mathrm{CD}^{+}$TILs in human OvCa. The expression of B7S1 in the TME was strongly correlated with poor $\mathrm{CD}^{+} \mathrm{T}$-cell responses. Functional enrichment and GSEA analyses illustrated that VTCN1 was significantly involved in T-cell regulation, cancerrelated pathways, and hallmarks in OvCa. These results indicated that B7S1 could serve as a novel biomarker for diagnosis and could be considered as a potential immunotherapeutic target.

High expression of B7S1 in OvCa has been described, and the overexpression of $\mathrm{B} 7 \mathrm{~S} 1$ has been identified to be associated with tumor stage (Liang et al., 2016) and a worse progression-free survival (Ye et al., 2018). In our study, we further demonstrated robust expression of B7S1 in human OvCa. However, VTCN1 expression was not significantly correlated with tumor grade and disease-free survival, an observation not consistent with protein levels, suggesting that it may be related to the mechanism of posttranscriptional regulation. In contrast to a previous study showing that $\mathrm{B} 7 \mathrm{~S} 1$ is predominantly expressed by tumor cells, we found that $\mathrm{B} 7 \mathrm{~S} 1$ could be detected in both $\mathrm{CD} 45^{-}$and $\mathrm{CD} 45^{+}$ 

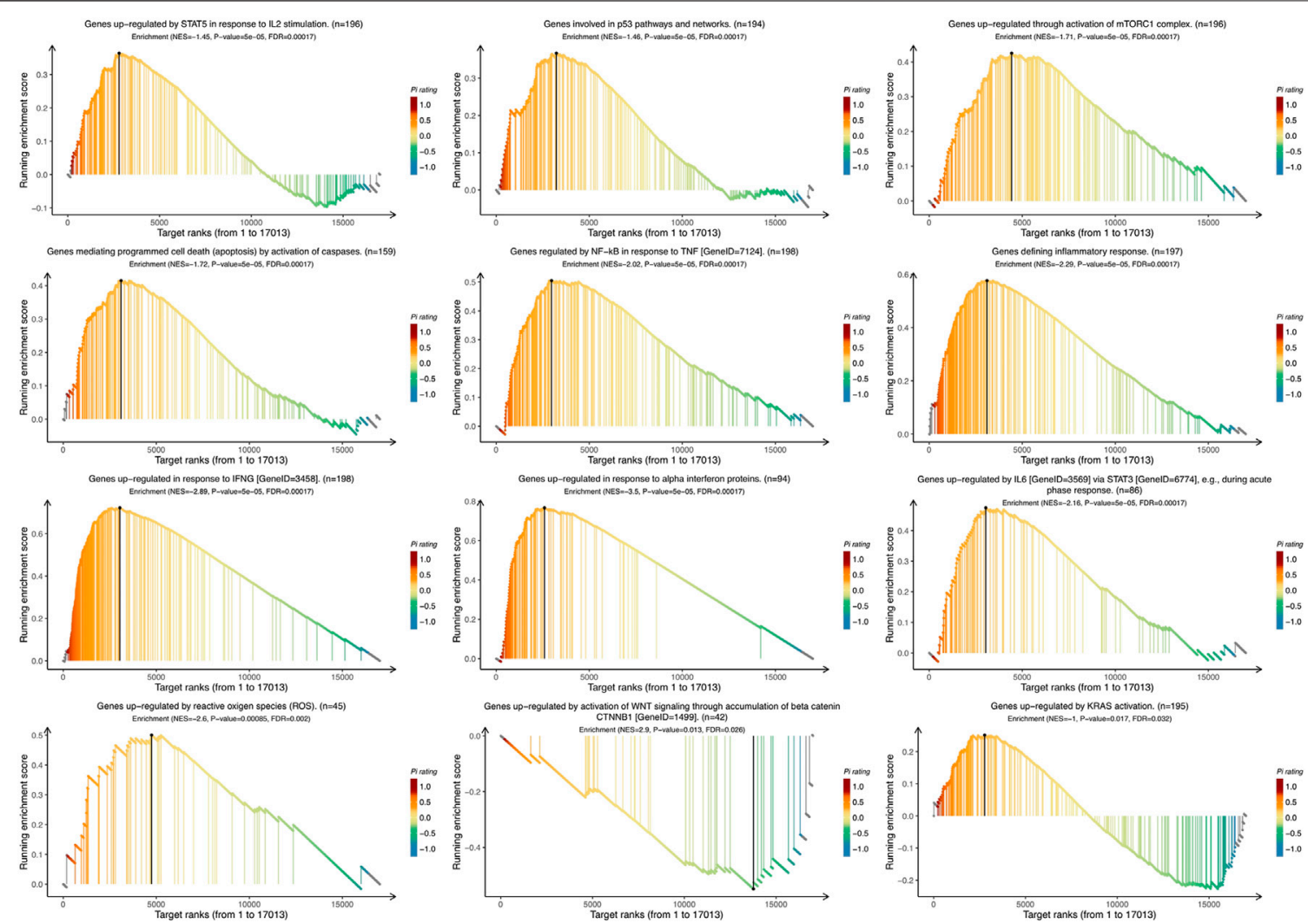

FIGURE 5 | The Gene Set Enrichment Analysis (GSEA) of the relationship between the expression level of VTCN1 in the TCGA ovarian cancer dataset. The most involved significant hallmark pathways which were closely correlated with VTCN1 in OvCa obtained by GSEA. NES: normalized enrichment score; FDR: false discovery rate.

cells in the OvCa TME. In CD45 ${ }^{+}$cells, B7S1 was mainly detected within the $\mathrm{CD}^{+} 8^{+}$myeloid compartment, particularly mDCs, $\mathrm{Mø/Mo}$, and mMDSCs. Moreover, the expression levels of B7S1 in tumor sections and ascites were significantly higher than those in PBMCs. According to previous reports (Motzer et al., 2015; Patel and Kurzrock, 2015; Yi et al., 2018; Cai et al., 2020), high PD-L1 expression in the TME is favorably correlated with increased response rates and clinical benefits in PD-L1/PD-1 blockade therapies. However, in OvCa, PD-L1 is observed rather low expressed on tumor cells, and the percentage of PD-L1expressing APCs is not high in tumor sections or ascites (5). The low expression levels of PD-L1 may contribute to the low overall response rates in patients with OvCa after anti-PD-L1/PD-1 therapies. The high levels of B7S1 in the TME and the distinct expression patterns from PD-L1 indicate that B7S1 may be a potential therapeutic target in patients with $\mathrm{OvCa}$ who are insensitive to PD-L1/PD-1 inhibition.

The expression level of B7S1 is negatively correlated with the density of TILs (Wang and Wang, 2020). In our study, compared with $\mathrm{B} 7 \mathrm{~S} 1^{\text {hi }}$ patients, $\mathrm{B} 7 \mathrm{~S} 1^{\text {low }}$ patients displayed decreased frequencies of $\mathrm{CD}^{+} \mathrm{T}$ cells and $\mathrm{CD} 4^{+} \mathrm{Foxp}^{+}$cells, but increased fractions of $\mathrm{CD}^{+}$TILs and $\mathrm{CD} 8 / \mathrm{CD} 4$ ratios. In hepatocellular carcinoma, B7S1 has been found to be upregulated in APCs and to be related to T-cell exhaustion via its receptor expressed on early activated $\mathrm{CD}^{+}$TILs. B7S1 blockade was found to promote $\mathrm{CD}^{+} \mathrm{T}$ cell-mediated antitumor immunity in a murine cancer model (Li et al., 2018). Consistent with this, in OvCa, pB7S1R has been detected in $\mathrm{CD}^{+} \mathrm{T}, \mathrm{CD}^{+} \mathrm{T}, \mathrm{NK}$, and NK T cells. The expression patterns of $\mathrm{B} 7 \mathrm{~S} 1$ and $\mathrm{pB} 7 \mathrm{~S} 1 \mathrm{R}$ strongly suggest that B7S1 has an important role in regulating $\mathrm{T}$ cells in antitumor immunity in OvCa. Indeed, compared with $\mathrm{B} 7 \mathrm{~S} 1^{\text {low }}$ patients, $\mathrm{B} 7 \mathrm{~S} 1^{\text {hi }}$ patients displayed more severe immunosuppression in the TME, with a higher percentage of regulatory $\mathrm{T}$ cells and a lower fraction of $\mathrm{CD}^{+}$TILs. Moreover, $\mathrm{CD}^{+}$TILs isolated from $\mathrm{B} \mathrm{S} 1^{\text {hi }}$ patients displayed characteristic exhausted T-cell phenotypes, including PD-1 expression, decreased CD27 and Ki67 expression, and TNF- $\alpha$ secretion. Thus, through crosspresentation between APCs and $\mathrm{CD}^{+} \mathrm{T}$ cells, B7S1 may facilitate the suppression of antitumor immunity in OvCa.

Using publicly available clinical data from TCGA, we focused on the biological functions and underlying mechanisms of VTCN1 in the TME via GO enrichment analysis and GSEA. Several biological processes correlated with immune regulation were identified, including regulation of leukocyte activation, regulation of lymphocyte activation, antigen processing and presentation, regulation of leukocyte cell-cell adhesion, and response to IFN- $\gamma$. Importantly, B7S1 can be detected on 
APCs isolated from OvCa, and high expression of B7S1 is associated with decreased cytotoxic activity of $\mathrm{CD}^{+}$TILs. Moreover, B7S1 has been reported to inhibit the activation and function of $\mathrm{T}$ cells, potently suppressing the proliferation, cytokine production, and cytotoxicity of activated $\mathrm{T}$ cells (Sica et al., 2003). When evaluating the underlying mechanisms of VTCN1, we found that VTCN1 was significantly involved in most significant hallmark pathways, including IL-2/STAT5 signaling, p53 pathway, mTORC1 signaling, apoptosis, TNF-a signaling via NF- $\kappa B$, inflammatory response, IFN- $\gamma$ response, IFN- $\alpha$ response, IL-6/JAK/STAT3 signaling, reactive oxygen species signaling, $\mathrm{WNT} / \beta$-catenin signaling, and KRAS signaling, in OvCa. These hallmark pathways are related to inflammation, immune regulation, tumor suppressor mutations, reactive oxygen species, cell cycle regulation, and apoptosis. Thus, our findings highlighted the specific associations that may trigger carcinogenesis.

Taken together, our results showed that overexpression of B7S1 in OvCa was positively correlated with antitumor immunosuppression. We also identified a potential immunotherapeutic target for OvCa. Further studies are needed to confirm these results and elucidate the underlying mechanisms.

\section{DATA AVAILABILITY STATEMENT}

The datasets presented in this study can be found in online repositories. The names of the repository/repositories and

\section{REFERENCES}

Bray, F., Ferlay, J., Soerjomataram, I., Siegel, R. L., Torre, L. A., and Jemal, A. (2018). Global Cancer Statistics 2018: GLOBOCAN Estimates of Incidence and Mortality Worldwide for 36 Cancers in 185 Countries. CA Cancer J. Clin. 68 (6), 394-424. doi:10.3322/caac.21492

Cai, D., Li, J., Liu, D., Hong, S., Qiao, Q., Sun, Q., et al. (2020). Tumor-expressed B7-H3 Mediates the Inhibition of Antitumor T-Cell Functions in Ovarian Cancer Insensitive to PD-1 Blockade Therapy. Cell Mol Immunol 17 (3), 227-236. doi:10.1038/s41423-019-0305-2

Capriglione, S., Luvero, D., Plotti, F., Terranova, C., Montera, R., Scaletta, G., et al. (2017). Ovarian Cancer Recurrence and Early Detection: May HE4 Play a Key Role in This Open challenge? A Systematic Review of Literature. Med. Oncol. 34 (9), 164. doi:10.1007/s12032-017-1026-y

Chen, G. M., Kannan, L., Geistlinger, L., Kofia, V., Safikhani, Z., Gendoo, D. M. A., et al. (2018). Consensus on Molecular Subtypes of High-Grade Serous Ovarian Carcinoma. Clin. Cancer Res. 24 (20), 5037-5047. doi:10.1158/1078-0432.CCR18-0784

Chen, X., Wang, L., Wang, W., Zhao, L., and Shan, B. (2016). B7-H4 Facilitates Proliferation of Esophageal Squamous Cell Carcinoma Cells through Promoting Interleukin-6/signal Transducer and Activator of Transcription 3 Pathway Activation. Cancer Sci. 107 (7), 944-954. doi:10.1111/cas.12949

Choi, I. H., Zhu, G., Sica, G. L., Strome, S. E., Cheville, J. C., Lau, J. S., et al. (2003). Genomic Organization and Expression Analysis of B7-H4, an Immune Inhibitory Molecule of the B7 Family. J. Immunol. 171 (9), 4650-4654. doi:10.4049/jimmunol.171.9.4650

Disis, M. L. P. M., S. P., E. P. H., A. C. L., and Avelumab, al. e. (0010). Anti- PD-L1) in Patients with Recurrent/refractory Ovarian Cancer from the JAVELIN Solid Tumor Phase Ib Trial: Safety and Clinical Activity. J. Clin. Oncol. 2016 (34). doi:10.1016/j.immuni.2016.05.001 accession number(s) can be found in the article/ Supplementary Material.

\section{ETHICS STATEMENT}

The studies involving human participants were reviewed and approved by Ethical Committee of the Shanghai First Maternity and Infant Hospital (IEC approval NO. 2017-100). The patients/ participants provided their written informed consent to participate in this study.

\section{AUTHOR CONTRIBUTIONS}

DC performed the experiments. CW conducted the bioinformatic analysis. FW supervised and collected clinical specimens. DC, FW, and CW wrote and revised the article. LJ supervised the study.

\section{FUNDING}

This project was funded in part by the National Natural Science Foundation of China (82071653, 81730039,81671460), the National Key Research and Development Program of China (2017YFC1001401), and Shanghai Municipal Medical and Health Discipline Construction Projects (2017ZZ02015) to LJ.

Gaillard, S. L., Secord, A. A., and Monk, B. (2016). The Role of Immune Checkpoint Inhibition in the Treatment of Ovarian Cancer. Gynecol. Oncol. Res. Pract. 3, 11 doi:10.1186/s40661-016-0033-6

Hamanishi, J., Mandai, M., Iwasaki, M., Okazaki, T., Tanaka, Y., Yamaguchi, K., et al. (2007). Programmed Cell Death 1 Ligand 1 and Tumor-Infiltrating CD8+ T Lymphocytes Are Prognostic Factors of Human Ovarian Cancer. Proc. Natl. Acad. Sci. U S A. 104 (9), 3360-3365. doi:10.1073/pnas.0611533104

Hansen, J. D., Du Pasquier, L., Lefranc, M. P., Lopez, V., Benmansour, A., and Boudinot, P. (2009). The B7 Family of Immunoregulatory Receptors: a Comparative and Evolutionary Perspective. Mol. Immunol. 46 (3), 457-472. doi:10.1016/j.molimm.2008.10.007

Hong, S., Yuan, Q., Xia, H., Zhu, G., Feng, Y., Wang, Q., et al. (2019). Analysis of VISTA Expression and Function in Renal Cell Carcinoma Highlights VISTA as a Potential Target for Immunotherapy. Protein Cell 10 (11), 840-845. doi:10.1007/s13238-019-0642-z

Hung, H. I., Klein, O. J., Peterson, S. W., Rokosh, S. R., Osseiran, S., Nowell, N. H., et al. (2016). PLGA Nanoparticle Encapsulation Reduces Toxicity while Retaining the Therapeutic Efficacy of EtNBS-PDT In Vitro. Sci. Rep. 6, 33234. doi:10.1038/srep33234

Hwang, W. T., Adams, S. F., Tahirovic, E., Hagemann, I. S., and Coukos, G. (2012). Prognostic Significance of Tumor-Infiltrating T Cells in Ovarian Cancer: a Meta-Analysis. Gynecol. Oncol. 124 (2), 192-198. doi:10.1016/ j.ygyno.2011.09.039

Kang, F. B., Wang, L., Sun, D. X., Li, H. J., Li, D., Wang, Y., et al. (2017). B7-H4 Overexpression Is Essential for Early Hepatocellular Carcinoma Progression and Recurrence. Oncotarget 8 (46), 80878-80888. doi:10.18632/ oncotarget. 20718

Krambeck, A. E., Thompson, R. H., Dong, H., Lohse, C. M., Park, E. S., Kuntz, S. M., et al. (2006). B7-H4 Expression in Renal Cell Carcinoma and Tumor Vasculature: Associations with Cancer Progression and Survival. Proc. Natl. Acad. Sci. U S A. 103 (27), 10391-10396. doi:10.1073/pnas.0600937103 
Kryczek, I., Wei, S., Zhu, G., Myers, L., Mottram, P., Cheng, P., et al. (2007). Relationship between B7-H4, Regulatory T Cells, and Patient Outcome in Human Ovarian Carcinoma. Cancer Res. 67 (18), 8900-8905. doi:10.1158/ 0008-5472.CAN-07-1866

Kryczek, I., Zou, L., Rodriguez, P., Zhu, G., Wei, S., Mottram, P., et al. (2006). B7H4 Expression Identifies a Novel Suppressive Macrophage Population in Human Ovarian Carcinoma. J. Exp. Med. 203 (4), 871-881. doi:10.1084/ jem.20050930

Li, J., Lee, Y., Li, Y., Jiang, Y., Lu, H., Zang, W., et al. (2018). Co-inhibitory Molecule B7 Superfamily Member 1 Expressed by Tumor-Infiltrating Myeloid Cells Induces Dysfunction of Anti-tumor CD8(+) T Cells. Immunity 48 (4), 773-786.e5. doi:10.1016/j.immuni.2018.03.018

Liang, L., Jiang, Y., Chen, J. S., Niu, N., Piao, J., Ning, J., et al. (2016). B7-H4 Expression in Ovarian Serous Carcinoma: a Study of 306 Cases. Hum. Pathol. 57, 1-6. doi:10.1016/j.humpath.2016.06.011

MacGregor, H. L., Garcia-Batres, C., Sayad, A., Elia, A., Berman, H. K., Toker, A., et al. (2019). Tumor Cell Expression of B7-H4 Correlates with Higher Frequencies of Tumor-Infiltrating APCs and Higher CXCL17 Expression in Human Epithelial Ovarian Cancer. Oncoimmunology 8 (12), el665460. doi:10.1080/2162402X.2019.1665460

Mariathasan, S., Turley, S. J., Nickles, D., Castiglioni, A., Yuen, K., Wang, Y., et al. (2018). TGFbeta Attenuates Tumour Response to PD-L1 Blockade by Contributing to Exclusion of $\mathrm{T}$ Cells. Nature 554 (7693), 544-548. doi:10.1038/nature25501

McDermott, D. F., Sosman, J. A., Sznol, M., Massard, C., Gordon, M. S., Hamid, O., et al. (2016). Atezolizumab, an Anti-programmed Death-Ligand 1 Antibody, in Metastatic Renal Cell Carcinoma: Long-Term Safety, Clinical Activity, and Immune Correlates from a Phase Ia Study. J. Clin. Oncol. 34 (8), 833-842. doi:10.1200/JCO.2015.63.7421

Motzer, R. J., Rini, B. I., McDermott, D. F., Redman, B. G., Kuzel, T. M., Harrison, M. R., et al. (2015). Nivolumab for Metastatic Renal Cell Carcinoma: Results of a Randomized Phase II Trial. J. Clin. Oncol. 33 (13), 1430-1437. doi:10.1200/ JCO.2014.59.0703

Pardoll, D. M. (2012). The Blockade of Immune Checkpoints in Cancer Immunotherapy. Nat. Rev. Cancer 12 (4), 252-264. doi:10.1038/nrc3239

Patel, S. P., and Kurzrock, R. (2015). PD-L1 Expression as a Predictive Biomarker in Cancer Immunotherapy. Mol. Cancer Ther. 14 (4), 847-856. doi:10.1158/ 1535-7163.MCT-14-0983

Peng, H. X., Wu, W. Q., Yang, D. M., Jing, R., Li, J., Zhou, F. L., et al. (2015). Role of B7-H4 siRNA in Proliferation, Migration, and Invasion of LOVO Colorectal Carcinoma Cell Line. Biomed. Res. Int. 2015, 326981. doi:10.1155/2015/326981

Quandt, D., Fiedler, E., Boettcher, D., Marsch, W., and Seliger, B. (2011). B7-h4 Expression in Human Melanoma: its Association with Patients' Survival and Antitumor Immune Response. Clin. Cancer Res. 17 (10), 3100-3111. doi:10.1158/1078-0432.CCR-10-2268

Ru, B., Wong, C. N., Tong, Y., Zhong, J. Y., Zhong, S. S. W., Wu, W. C., et al. (2019). TISIDB: an Integrated Repository portal for Tumor-Immune System Interactions. Bioinformatics 35 (20), 4200-4202. doi:10.1093/bioinformatics/btz210

Sica, G. L., Choi, I. H., Zhu, G., Tamada, K., Wang, S. D., Tamura, H., et al. (2003). B7-H4, a Molecule of the B7 Family, Negatively Regulates T Cell Immunity. Immunity 18 (6), 849-861. doi:10.1016/s1074-7613(03)00152-3

Sun, C., Mezzadra, R., and Schumacher, T. N. (2018). Regulation and Function of the PD-L1 Checkpoint. Immunity 48 (3), 434-452. doi:10.1016/ j.immuni.2018.03.014
Sun, Y., Wang, Y., Zhao, J., Gu, M., Giscombe, R., Lefvert, A. K., et al. (2006). B7H3 and B7-H4 Expression in Non-small-cell Lung Cancer. Lung Cancer 53 (2), 143-151. doi:10.1016/j.lungcan.2006.05.012

Tang, Z., Li, C., Kang, B., Gao, G., Li, C., and Zhang, Z. (2017). GEPIA: a Web Server for Cancer and normal Gene Expression Profiling and Interactive Analyses. Nucleic Acids Res. 45 (W1), W98-W102. doi:10.1093/nar/gkx247

Thommen, D. S., Koelzer, V. H., Herzig, P., Roller, A., Trefny, M., Dimeloe, S., et al. (2018). A Transcriptionally and Functionally Distinct PD-1(+) CD8(+) T Cell Pool with Predictive Potential in Non-small-cell Lung Cancer Treated with PD1 Blockade. Nat. Med. 24 (7), 994-1004. doi:10.1038/s41591-018-0057-z

Tringler, B., Zhuo, S., Pilkington, G., Torkko, K. C., Singh, M., Lucia, M. S., et al. (2005). B7-h4 Is Highly Expressed in Ductal and Lobular Breast Cancer. Clin. Cancer Res. 11 (5), 1842-1848. doi:10.1158/1078-0432.CCR-04-1658

Wang, J. Y., and Wang, W. P. (2020). B7-H4, a Promising Target for Immunotherapy. Cell Immunol 347, 104008. doi:10.1016/ j.cellimm.2019.104008

Wang, L., Yang, C., Liu, X. B., Wang, L., and Kang, F. B. (2018). B7-H4 Overexpression Contributes to Poor Prognosis and Drug-Resistance in Triple-Negative Breast Cancer. Cancer Cel Int 18, 100. doi:10.1186/s12935018-0597-9

Wang, X., Hao, J., Metzger, D. L., Ao, Z., Chen, L., Ou, D., et al. (2012). B7-H4 Treatment of T Cells Inhibits ERK, JNK, P38, and AKT Activation. PLoS One 7 (1), e28232. doi:10.1371/journal.pone.0028232

Ye, Y., Wang, J. J., Li, S. L., Wang, S. Y., and Jing, F. H. (2018). Does B7-H4 Expression Correlate with Clinicopathologic Characteristics and Survival in Ovarian Cancer?: A Systematic Review and PRISMA-Compliant MetaAnalysis. Medicine (Baltimore) 97 (32), e11821. doi:10.1097/ MD.0000000000011821

Yi, M., Jiao, D., Xu, H., Liu, Q., Zhao, W., Han, X., et al. (2018). Biomarkers for Predicting Efficacy of PD-1/pd-L1 Inhibitors. Mol. Cancer 17 (1), 129. doi:10.1186/s12943-018-0864-3

Yu, G., Wang, L. G., Han, Y., and He, Q. Y. (2012). clusterProfiler: an R Package for Comparing Biological Themes Among Gene Clusters. OMICS 16 (5), 284-287. doi:10.1089/omi.2011.0118

Zhang, L., Conejo-Garcia, J. R., Katsaros, D., Gimotty, P. A., Massobrio, M., Regnani, G., et al. (2003). Intratumoral T Cells, Recurrence, and Survival in Epithelial Ovarian Cancer. N. Engl. J. Med. 348 (3), 203-213. doi:10.1056/ NEJMoa020177

Zhu, J., Chu, B. F., Yang, Y. P., Zhang, S. L., Zhuang, M., Lu, W. J., et al. (2013). B7H4 Expression Is Associated with Cancer Progression and Predicts Patient Survival in Human Thyroid Cancer. Asian Pac. J. Cancer Prev. 14 (5), 3011-3015. doi:10.7314/apjcp.2013.14.5.3011

Conflict of Interest: The authors declare that the research was conducted in the absence of any commercial or financial relationships that could be construed as a potential conflict of interest.

Copyright (c) 2021 Cai, Wang, Wang and Jin. This is an open-access article distributed under the terms of the Creative Commons Attribution License (CC $B Y$ ). The use, distribution or reproduction in other forums is permitted, provided the original author(s) and the copyright owner(s) are credited and that the original publication in this journal is cited, in accordance with accepted academic practice. No use, distribution or reproduction is permitted which does not comply with these terms. 